\section{A case of penile zip trap}

\author{
S Parthasarathy ${ }^{1}$, N S Radhakrishnan ${ }^{2}$, C Rajah $^{3}$
}

Sri Lanka Journal of Child Health 2010; 39: 70-71

(Key words: penile zip trap)

\begin{abstract}
A ten year old boy presented with a zip trapping the penis of two days duration. The zip was removed successfully under local anaesthesia. Various methods of removal of a zipper are described
\end{abstract}

\section{Case Report}

A 10 year old boy reported to Government District Headquarters Hospital, Kumbakonam, India with a history of trapping of the prepuce by a zip of two days duration. (Figure 1)

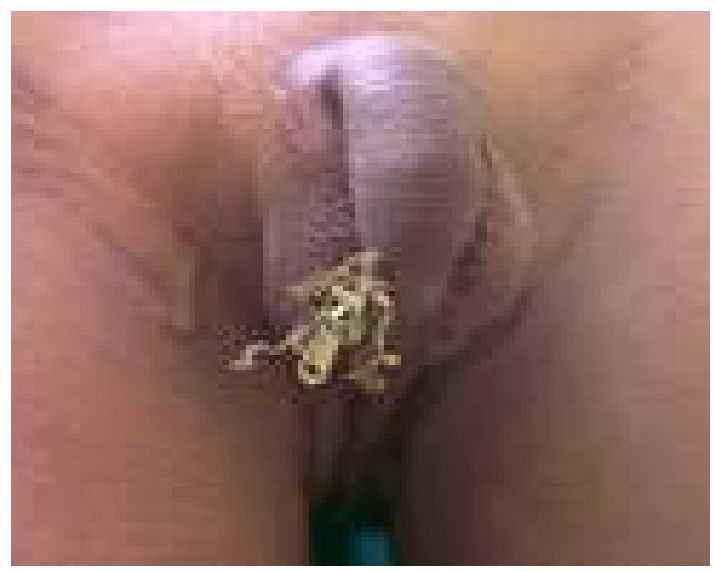

Figure 1 showing entrapment of penis in zipper

After a penile block, the end of the zipper was cut with scissors and the zipper was pulled in the opposite direction. It got released easily without any damage to the penile skin. There were no immediate post procedure complications.

${ }^{1}$ Chief Anaesthesiologist, ${ }^{2}$ Consultant Surgeon,

${ }^{3}$ Chief Paediatrician, Government District Headquarters Hospital, Kumbakonam, South India.

(Received on 23 February 2010. Accepted after revision on 19 March 2010)

\section{Discussion}

Entrapment of unprotected penis into zipper is the most common cause of preputial injury in children ${ }^{1}$. These children are often distressed and frightened due to several unsuccessful and painful attempts at extrication by the parents ${ }^{2}$.

Different management strategies are reported, including an aggressive approach of partial excision of the entrapped foreskin, or a circumcision under general anaesthesia ${ }^{2}$. The non aggressive management strategies involve some form of mechanical disengagement of the zipper from the skin ${ }^{2}$. One crude method is to push the zip slider in the opposite direction from which it had originally trapped the skin. However, tugging at the zipper in either direction may result in tighter entrapment or further bruising $^{2}$. Lateral compression of the zip using pliers sometimes frees the prepuce ${ }^{2}$. Another technique described is to unfasten the zipper one tooth at a time, alternating sides ${ }^{2}$. Also reported is giving a transverse cut through the cloth strips holding the tooth line, and allowing disengagement from both sides of the entrapment $^{2}$. Still others report use of mineral oil followed by traction ${ }^{3}$. However, the most common method described so far, involves the division of the median bar connecting the anterior and posterior faceplates of the zip slider, using a bone cutter or a mini hacksaw ${ }^{4}$ (figure 2 ).

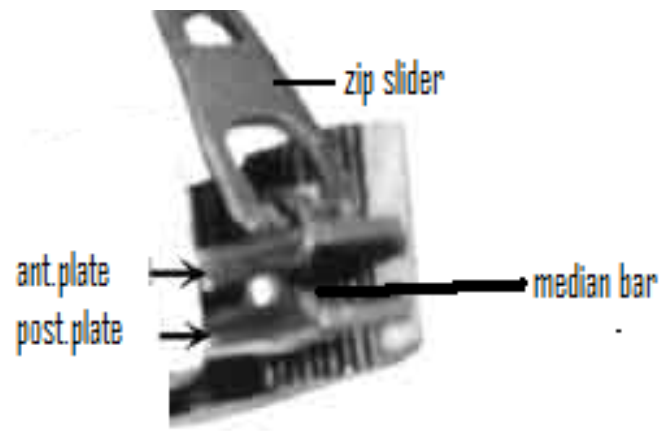

Figure $\overline{2}$ showing the various parts of a zipper 
There is one more described technique i.e. the thin blade of a small screwdriver can be inserted between the outer and inner faceplates of the zip slider and a twisting movement is made towards the median bar. This will widen the gap between faceplates and may help to disengage the prepuce ${ }^{5}$. These strategies of zip manipulation, while effective, are not very rapid and cause sufficient movement of the zipper and the penis and therefore, often require local anaesthesia. We used penile block in our case. Moreover, if the median bar lies deep or a portion of the entrapped prepuce projects ahead of the zip slider, it may be very difficult and potentially dangerous to divide the median bar using these methods. Hence, in such situations, the other options should be thought of. With such exhaustive description of various techniques we should admit that ours was a crude technique but was successful. We feel that our success was due to adequate anaesthesia and understanding of the zipper end trapping the prepuce. Hence, as soon the end was divided, the slider could be easily released. We suggest that comprehension of the mechanisms of zipper entrapment may avoid invasive therapies like circumcision. We propose that awareness about the importance of wearing briefs inside pantaloons (trousers) for all kids who are vulnerable to such events should be given to parents and teachers. We suggest that such common and easily manageable problems should be given top priority in the curriculum.

\section{Acknowledgements}

We are extremely thankful to Dr. T. Anbalagan, Hospital Superintendent for allowing us to publish the article.

\section{References:}

1. Yip A, Ng SK, Wong WC, et al. Injury to the prepuce. Br J Urol 1989; 63(5):535-8.

2. Mishra SC. Safe and painless manipulation of penile zipper entrapment. Indian Pediatr 2006; 43(3):252-4.

3. Kanegaye JT, Schonfeld N. Penile zip entrapment: A simple and less threatening approach using mineral oil. Pediatr Em Care 1993; 9: 90-91.

4. Strait RT. A novel method of removal of penile zipper entrapment. Pediatr Emerg Care 1999; 15: 412-3.

5. Raveenthiran V; Releasing of zipperentrapped foreskin: a novel nonsurgical technique. Pediatr Emerg Care 2007; 23(7): 463-4. 
